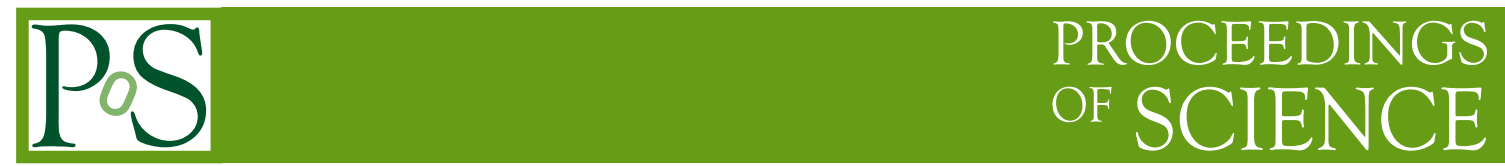

\title{
Overview on Exclusive Processes at HERMES
}

\section{Riccardo Fabbri *}

DESY, Notkestrasse 85, D-22607 Hamburg, Germany

E-mail: Riccardo.Fabbri@desy.de

Hard exclusive production in deep inelastic lepton scattering provides new access to the poorly known Generalized Parton Distributions (GPDs) of the nucleon. Different observables for hard exclusive production of photons and mesons have been measured by the HERMES experiment. Emphasis is given to recent results which help determine the total angular momentum of quarks in the proton.

International Workshop on Diffraction in High-Energy Physics -DIFFRACTION 2006 September 5-10 2006

Adamantas, Milos island, Greece

${ }^{*}$ On behalf of the HERMES Collaboration 


\section{Introduction}

The analysis of hard exclusive processes can be used to investigate the generalized parton distributions (GPD) [1,2]. From a formal point of view GPDs show a continuity in describing both inclusive and exclusive processes, the usual parton distributions being a kinematic limit of GPDs. Also, the elastic form factors are specific moments of GPDs. Strong interest in the formalism of GPDs has emerged after GPDs were found to offer the first possibility to reveal the total angular momentum carried by the quarks and gluons in the nucleon [2].

In this paper, the latest results obtained by the DESY experiment HERMES [3] on Deeply Virtual Compton Scattering (DVCS) and exclusive meson production are reviewed. The data presented here were collected using internal, longitudinally or transversely polarized or unpolarized hydrogen deuterium targets in the longitudinally $27.6 \mathrm{GeV}$ HERA positron (electron) storage ring.

\section{Deeply Virtual Compton Scattering}

The basic mechanism for DVCS is a quark absorbing a virtual photon (in electroproduction) and radiating a real photon. At large photon virtuality the process amplitude can be factorized into a convolution of a hard scattering term exactly calculable in perturbative QCD, and a soft (non perturbative) term [4]. The soft term describes the nucleon transition in the process, and is parameterized by the four leading-twist GPDs which conserve quark helicity, $H, E, \tilde{H}$, and $\tilde{E}$. The process appears to provide the theoretically cleanest access to GPDs compared to other competitive hard exclusive processes, whose amplitudes are described by additional non-perturbative terms. In hard exclusive processes, like DVCS, a direct extraction of the GPDs from experimentally measured observables cannot be in general performed because at the amplitude level they are convoluted, in a flavor sum, with the hard scattering term, and cannot be disentangled. As a consequence, models of GPDs are to be constructed to calculate observables that have to be compared to corresponding experimental results. The convoluted $H, E, \tilde{H}$, and $\tilde{E}$ are traditionally represented with the symbols $\mathscr{H}, \mathscr{E}, \tilde{\mathscr{H}}$, and $\tilde{\mathscr{E}}$, respectively.

Experimentally, for electroproduction of photons, one cannot disentangle the DVCS from the Bethe-Heitler (BH) process, where the virtual photon is absorbed by the nucleon target and a real photon is emitted by either the incoming or the outgoing lepton. Within the HERMES kinematical acceptance the DVCS cross section is at least one order of magnitude smaller than the BH one [5]. Nevertheless, the DVCS amplitude can be still accessed through the BH-DVCS interference term, which can be projected out by considering specific cross section asymmetries in the azimuthal angle $\phi$. The latter is defined as the angle between the incoming and outgoing lepton trajectories and the plane correspondingly defined by the virtual and the real photon.

The event selection at HERMES requires events with exactly one photon and one charged track, identified as the scattered lepton with $Q^{2}>1 \mathrm{GeV}^{2}$,where $-Q^{2}$ is the squared four-momentum of the initial virtual photon. The recoiling proton is not detected, and exclusive events are identified by requiring the missing mass $M_{X}$ of the reaction $e p \rightarrow e \gamma X$ to correspond to the proton mass. Due to the finite energy resolution the exclusive sample is selected in the region $-1.5<M_{X}<1.7 \mathrm{GeV}$, based on signal-to-background studies using Monte Carlo simulations [6].

For an unpolarized target, the beam-charge cross section asymmetry $A_{C}(\phi)$ with unpolarized lepton of either charge, and the beam-spin cross section asymmetry $A_{L U}(\phi)$ using a longitudinally 

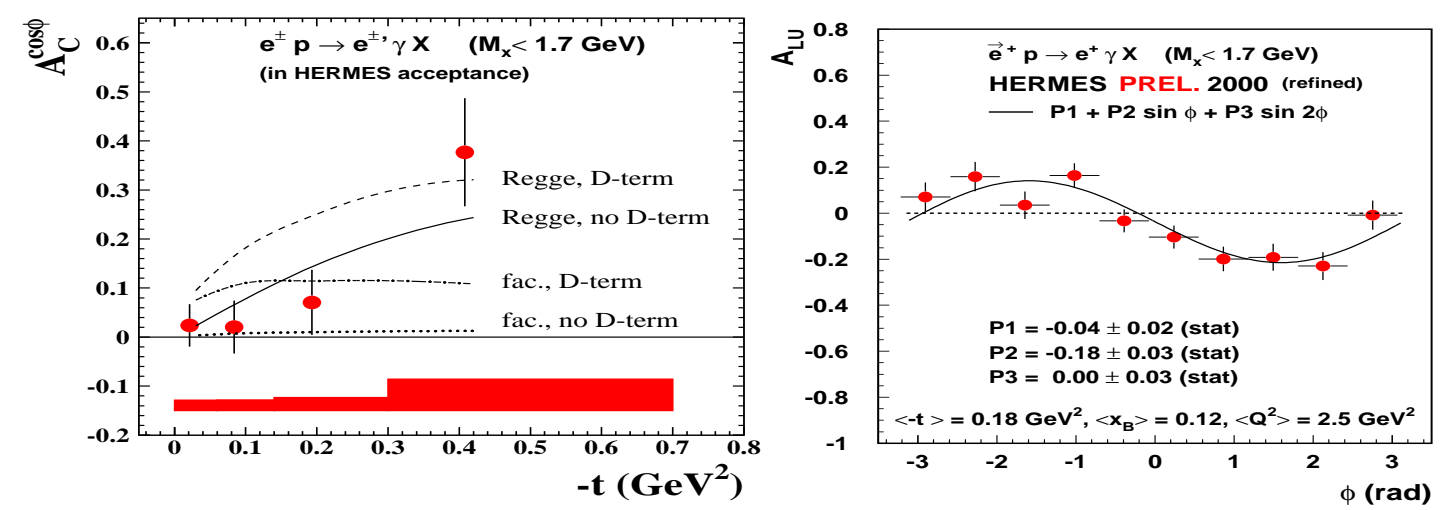

Figure 1: The Left panel: the $t$-dependence of $\cos \phi$ moment of beam-charge asymmetry $A_{C}$. Different predictions in the GPD framework are also shown. Right Panel: the $\phi$-dependence of the beam-spin asymmetry $A_{L U}$. Superimposed to the data are the fi t results which show the $\sin \phi$ modulation of $A_{L U}$.

polarized positron beam, at leading-twist in the HERMES kinematics reduce to [5]

$$
\begin{aligned}
& A_{C}(\phi) \stackrel{\text { def }}{=} \frac{d \sigma\left(e^{+} p, \phi\right)-d \sigma\left(e^{-} p, \phi\right)}{d \sigma\left(e^{+} p, \phi\right)+d \sigma\left(e^{-} p, \phi\right)} \sim \cos \phi \cdot \operatorname{Re}\left(F_{1} \mathscr{H}\right), \\
& A_{L U}(\phi) \stackrel{\text { def }}{=} \frac{d \sigma\left(\overrightarrow{e^{+}} p, \phi\right)-d \sigma\left(\overleftarrow{e^{+}} p, \phi\right)}{d \sigma\left(\overrightarrow{e^{+}} p, \phi\right)+d \sigma\left(e^{+} p, \phi\right)} \sim \sin \phi \cdot \operatorname{Im}\left(F_{1} \mathscr{H}\right),
\end{aligned}
$$

$F_{1}$ being the Dirac form factor of the proton.

The first measurement of the asymmetry $A_{C}$ was performed by HERMES using a proton target [7]. The dependence of the $\cos \phi$ moment on the squared four-momentum transfer $t$ to the target is shown in the left panel of Fig. 1, and compared with predictions in the GPD framework based on [8] (VGG) with different assumptions on the GPDs. The data seem to disfavor the model with the non-factorized $t$-dependence (Regge) for the GPDs with the contribution of the so-called $D$-term [9]. The $\phi$-dependence of the asymmetry $A_{L U}$ is shown in the right panel of Fig. 1. Also displayed are the fit results which show the expected leading-twist sin $\phi$ modulation of the asymmetry. The longitudinal target-spin cross section asymmetry $A_{U L}(\phi)$ for an unpolarized positron beam, at leading-twist in the HERMES kinematics, is expected to reduce to [10]

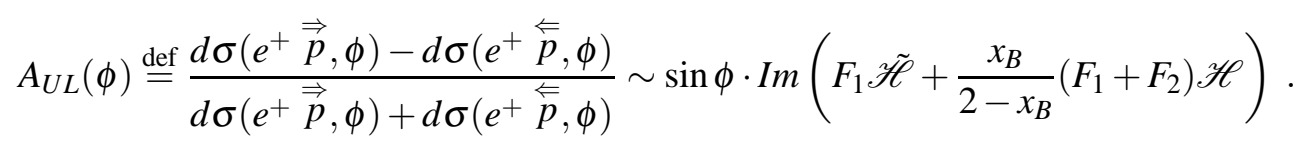

The $\phi$-dependence of the longitudinal target-spin asymmetry on a proton target is shown in the left panel of Fig. 2, where the fit results show the expected non-zero leading-twist $\sin \phi$ modulation of the asymmetry. The $\sin 2 \phi$ moment is $3 \sigma$ different from zero, an indication that higher-twist effects might contribute to $A_{U L}$ in the HERMES kinematics. A useful observable to access the convoluted GPDs $\mathscr{E}$ and $\tilde{\mathscr{E}}$ is the transverse target-spin asymmetry with unpolarized beam $A_{U T}$, which in the HERMES kinematic reads

$$
\begin{aligned}
A_{U T}\left(\phi-\phi_{S}\right) & \stackrel{\text { def }}{=} \frac{d \sigma\left(\phi-\phi_{S}\right)-d \sigma\left(\phi-\phi_{S}+\pi\right)}{d \sigma\left(\phi-\phi_{S}\right)+d \sigma\left(\phi-\phi_{S}+\pi\right)} \\
& \sim \operatorname{Im}\left[F_{2} \mathscr{H}-F_{1} \mathscr{E}\right] \cdot \sin \left(\phi-\phi_{S}\right) \cos \phi+\operatorname{Im}\left[F_{2} \tilde{\mathscr{H}}-F_{1} \frac{x_{B}}{2-x_{B}} \tilde{\mathscr{E}}\right] \cdot \cos \left(\phi-\phi_{S}\right) \sin \phi,
\end{aligned}
$$



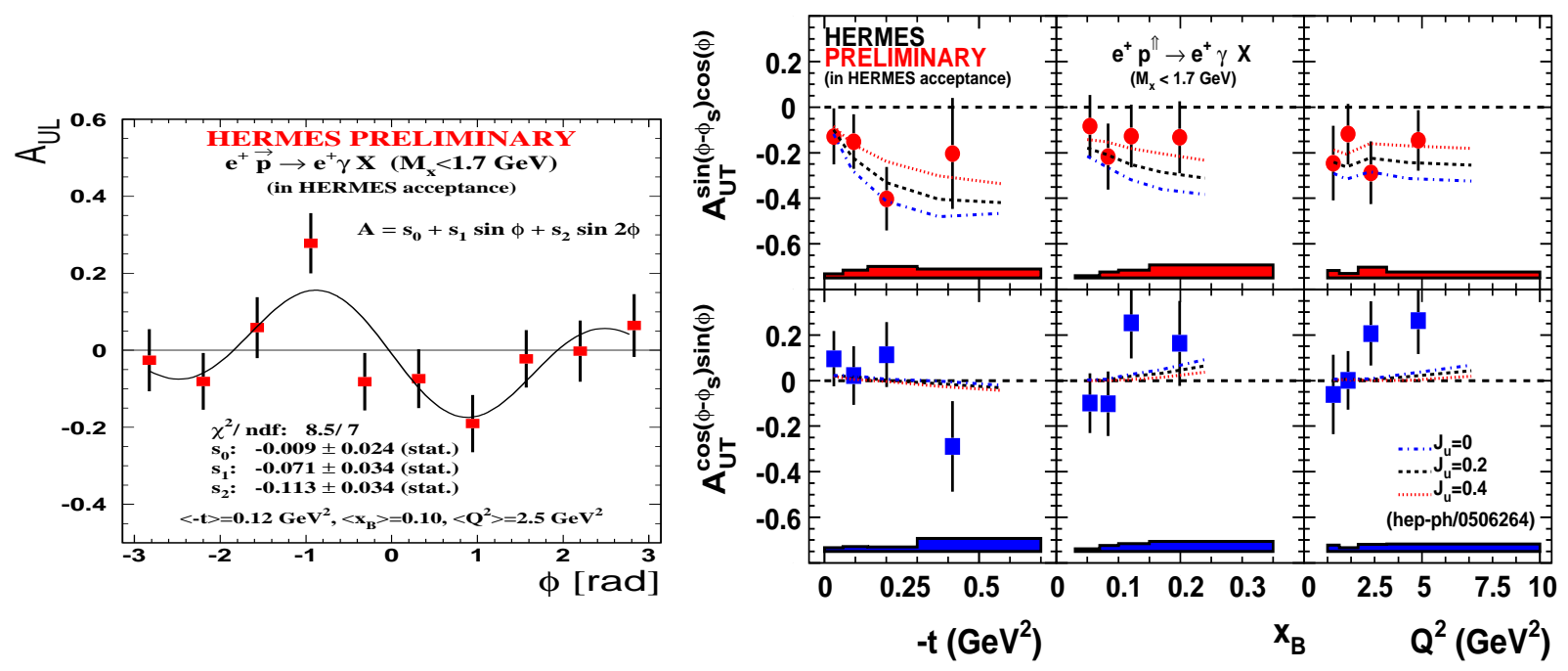

Figure 2: Left panel: the $\phi$-dependence of the asymmetry $A_{U L}$ on a proton target. The fit results show the non-zero leading-twist $\sin \phi$ and higher-twist $\sin 2 \phi$ modulations of the asymmetry. Right panel: the $t$-, Bjorken $x_{B^{-}}$, and $Q^{2}$-dependences of the moments $A_{U T}^{\sin \left(\phi-\phi_{S}\right) \cos \phi}$ (upper panels) and $A_{U T}^{\cos \left(\phi-\phi_{S}\right) \sin \phi}$ (bottom panels) on proton target. Also shown are different predictions [12] based on GPDs. The systematic uncertainty is given by the error band.

where $\phi_{S}$ denotes the azimuthal angle between the target polarization plane with respect to the lepton plane, and $F_{2}$ is the Pauli form factor of the proton.

The kinematical dependences of the moments $A_{U T}^{\sin \left(\phi-\phi_{S}\right) \cos \phi}$ and $A_{U T}^{\cos \left(\phi-\phi_{S}\right) \sin \phi}$ on transversely polarized proton target are shown in the right panel of Fig. 2 [11]. Also shown are the predictions [12] based on the VGG code [8] with different ansaetze for the parametrisations of GPDs [13]. The displayed curves represent the moments evaluated for a set of $u$-quark total angular momentum values, $J_{u}$, as a model parameter for $E$, and for a fixed value of the $d$-quark total angular momentum $J_{d}=0$, as inspired by the results of recent lattice calculations [14]. Minor sensitivity was found to changes in the other parameters.

By comparing, over the all kinematic bins $i$, the measured moment and the moment predicted for different $J_{u}$ and $J_{d}$ assumptions (releasing the condition $J_{d}=0$ ) using the VGG code, the quantity $\Delta \chi^{2}=\chi^{2}-\chi_{\text {minimum }}^{2}$ is calculated from

$$
\chi^{2}\left(J_{u}, J_{d}\right)=\sum_{i}^{\text {kin bins }} \frac{\left[\left.A_{U T, i}^{\sin \left(\phi-\phi_{S}\right) \cdot \cos (\phi)}\right|_{\text {exp }}-\left.A_{U T, i}^{\sin \left(\phi-\phi_{S}\right) \cdot \cos (\phi)}\right|_{V G G\left(J_{u}, J_{d}\right)}\right]^{2}}{\delta A_{\text {stat }, i}^{2}+\delta A_{\text {syst }, i}^{2}+\delta A_{\text {accept }, i}^{2}} .
$$

The area in the $\left(J_{u}, J_{d}\right)$-plane, in which $\Delta \chi^{2}$ value is not larger than one is defined as the onestandard-deviation constraint on $J_{u}$ versus $J_{d}$, and is shown in Fig. 3 [15]. This HERMES result, described by the relation $J_{u}+J_{d} / 2.9=0.42 \pm 0.21 \pm 0.06$, provides the first GPD model-dependent constraint on the total angular momenta $J_{u}$ and $J_{d}$.

\section{Exclusive Meson Production}

An alternative process to access GPDs is the production of mesons by hard longitudinal virtual photons. In the Bjorken limit the process amplitude factorizes into a convolution similar to the 


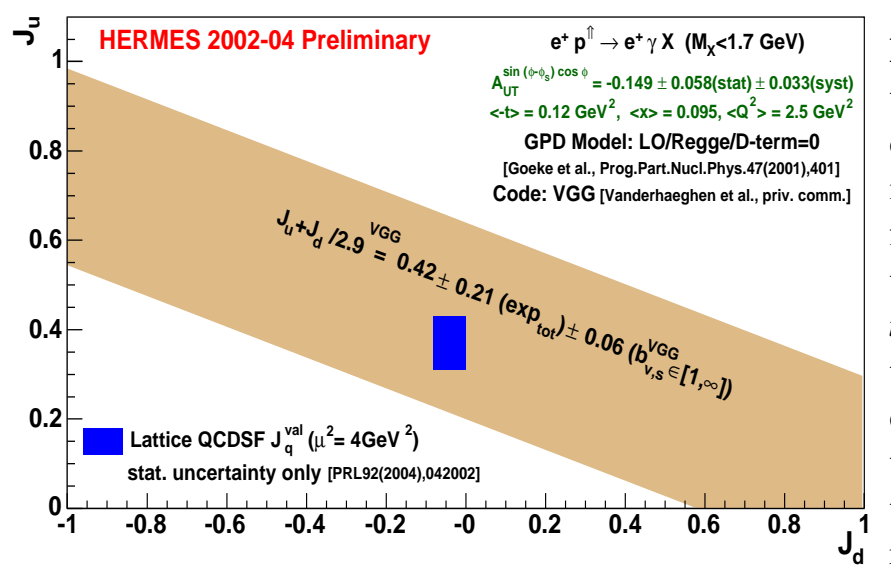

Figure 3: Model-dependent constraint on the total angular momentum $J_{u}$ vs $J_{d}$, obtained by comparing the experimental result and theoretical predictions on the moment $A_{U T}^{\sin \left(\phi-\phi_{S}\right) \cos \phi}$ for transverse target-spin asymmetry on a proton target and unpolarized positron beam. The $t$-dependence of the GPDs is modeled using the Regge ansatz [13]. The impact of this choice, compared to its alternatives, was found to be negligible [12]. The $D$-term contribution to the GPDs is set to zero, as suggested by the results on the beam-spin asymmetry.

DVCS case, with one additional soft part describing the hadronization of virtual partons into final meson state [16]. The factorization has been demonstrated only for longitudinal photons. Nevertheless, compared to the longitudinal case the cross section for transversely polarized photons was shown to be suppressed by a factor $1 / Q^{2}$.

While the leading order amplitude factorization holds, interesting selection rules among produced mesons and probed GPDs in the target nucleon arise, due to quantum numbers conservation in the QED and QCD processes involved [16]. Longitudinally polarized vector meson channels are sensitive only to the unpolarized GPDs $\left(H\right.$ and $E$ ), while the pseudo-scalar channels (e.g. $\left.\pi^{+}\right)$are sensitive only to the polarized GPDs $(\tilde{H}$ and $\tilde{E})$, without the need for a polarized target or beam. Additionally, flavor singlet and non-singlet combinations of unpolarized GPDs can be separately accessed by measuring either vector mesons or meson states with quantum numbers of the $f$ meson family [17]. In contrast, DVCS depends at the same time on both unpolarized and polarized GPDs.

\subsection{Hard Exclusive $\pi^{+}$Meson Production}

Exclusive $\pi^{+}$events were identified by detecting the scattered positron and the produced pion. Due to the limited experimental missing mass resolution, the exclusive reaction $e p \rightarrow e n \pi^{+}$cannot be separated unambiguously from neighboring channels which can be smeared into the exclusive region. Therefore the process $e p \rightarrow e \pi^{-} X$ was used to subtract the analyzed exclusive channel [18]. Indeed, the exclusive production of $\pi^{-}$on hydrogen target with a single recoiling nucleon in the final state is forbidden due to charge conservation.

The $Q^{2}$ dependence of the total cross section for three different Bjorken $x$ ranges is shown in

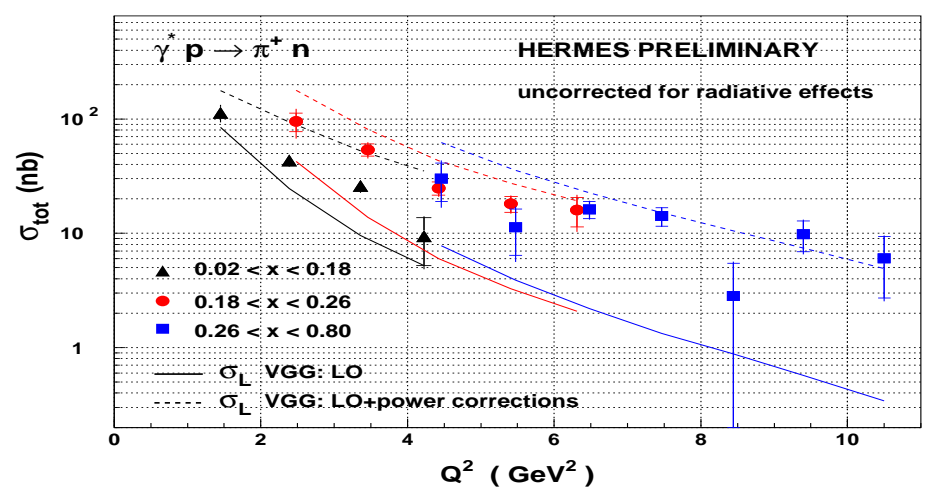

Figure 4: Total cross section for exclusive $\pi^{+}$production as function of $Q^{2}$ for three different $x$ ranges and integrated over $t$. The inner error bars represent the statistical uncertainty and the outer error bars the quadratic sum of statistical and systematic uncertainty. The curves represent calculations based on a GPD model [19]. 
Fig. 4. These preliminary data have not yet been corrected for radiative effects, roughly estimated to be about $20 \%$, and expected to show little dependence on $x$ or $Q^{2}$. The data are compared to calculations for the longitudinal part of the cross section computed within a GPD model [19]. At HERMES the separation of the transverse and longitudinal component of the cross section is not feasible. However, the data at larger $Q^{2}$ are expected to be dominated by the longitudinal part at HERMES kinematics. The $Q^{2}$ dependence is qualitatively in agreement with theoretical expectations. While the leading-order calculations (full lines) underestimate the data, the evaluation of the power corrections (dashed lines) appears too large.

\subsection{Hard Exclusive $\pi^{+} \pi^{-}$Production}

Hard exclusive pion pair production may involve both resonant and non-resonant channels mainly through the quark exchange mechanism which is dominant at HERMES kinematics. The pion pairs can be generated with the values of the strong isospin $I$, total angular momentum $J$, and $C$-parity of either a $\rho$-meson $(I=1, J=1,3 \ldots, C=-1)$, or an $f$-meson $(I=0, J=0,2 \ldots, C=+1)$. The quark exchange with $C=+1$ and $C=-1$ is described by flavor singlet and non-singlet $\mathscr{H}$ and $\mathscr{E}$ combinations [17], respectively. The latter cannot be accessed by analyzing $\rho^{0}$ decay. The interference between the two isospin channels provide information on the weaker isoscalar channel at the amplitude level.

For the purpose of studying the interference between $\pi^{+} \pi^{-}$production in $P$-wave $(I=1)$ and $S, D$-wave states $(I=0)$, the Legendre moment $\left\langle P_{1}(\cos \theta)\right\rangle[21]$ is particularly useful because it is only sensitive to such interference. Here $\theta$ is the polar angle of the $\pi^{+}$meson with respect to the direction of the $\pi^{+} \pi^{-}$pair in the pions rest-frame, and $P_{1}$ is the first-order Legendre polynomial.

The first experimental data for hard exclusive $\pi^{+} \pi^{-}$pair production on hydrogen and deuterium targets has been reported by HERMES [20]. The Bjorken $x$ dependence of $\left\langle P_{1}\right\rangle$ is shown in Fig. 5 for both targets in two regions of the $\pi^{+} \pi^{-}$invariant mass $m_{\pi \pi}$. In both $m_{\pi \pi}$ regions and for both targets, $\left\langle P_{1}\right\rangle$ is non-zero, which we interpret as originating from the interference of the resonant $\rho^{0} P$-wave with non-resonant $S$-wave $\pi^{+} \pi^{-}$production. The moment increases in magnitude with $x$, suggesting that the exchange of flavor non-singlet quark GPD combinations $(C=-1)$ becomes competitive with the dominant singlet exchange $(C=+1)$. Predictions for hydrogen in the GPD framework [21] are compared with the data, and are found to be in fair agreement with them.

\section{Conclusions}

Several observables in DVCS, hard exclusive $\pi^{+}$and $\pi^{+} \pi^{-}$production have been measured, and compared with GPD-based predictions in order to provide constraints on GPDs. In this regard, the first GPD model-dependent constraint on the total angular momenta $J_{u}$ and $J_{d}$ has been obtained.

\section{References}

[1] D. Müller et al., Fortsch. Phys. 42 (1994) 101; A. V. Radyushkin, Phys. Rev. D 56 (1997) 5524.

[2] X. Ji, J. Phys. G 24 (1998) 1181. 

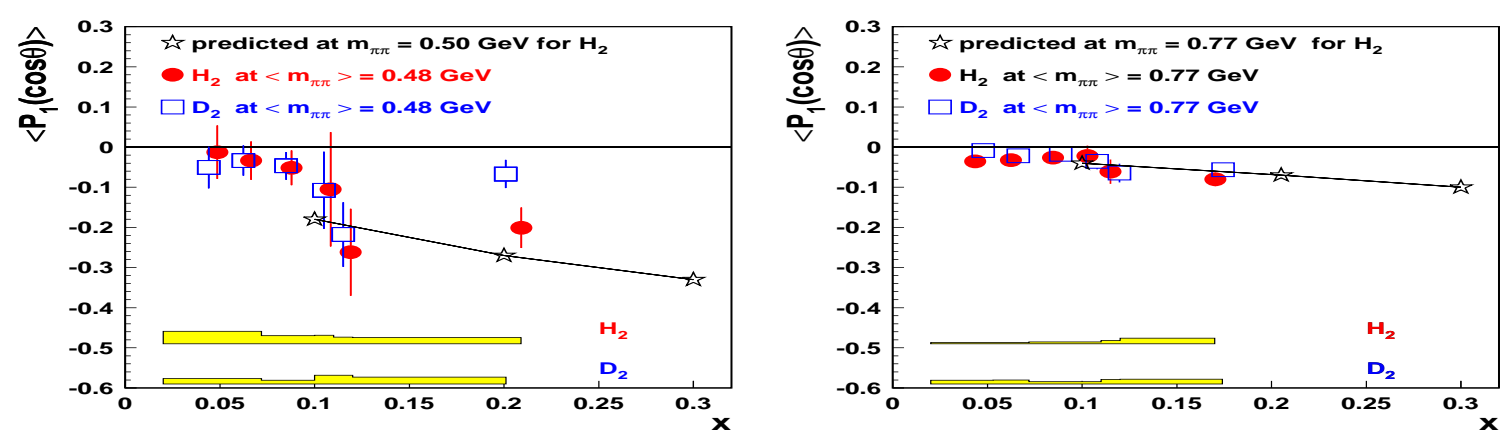

Figure 5: The $x$-dependence of the Legendre moment $\left\langle P_{1}\right\rangle$ for both hydrogen and deuterium targets, in the regions $0.30<m_{\pi \pi}<0.60 \mathrm{GeV}$ (left panel) and $0.60<m_{\pi \pi}<0.95 \mathrm{GeV}$ (right panel), at $\left\langle Q^{2}\right\rangle \sim 2 \mathrm{GeV}^{2}$. The systematic uncertainty is given by the error band. Theoretical predictions (stars) from [21] for hydrogen, which neglect two-gluon exchange mechanism, are compared with the data.

[3] HERMES Coll., K. Ackerstaff et al., Nucl. Inst. and Methods A 417 (1998) 230.

[4] A. V. Radyushkin, Phys. Lett. B 380 (1996) 417.; X. Ji, Phys. Rev. D 55 (1997) 7114.

[5] V. A. Korotkov and W.-D. Novak, Eur. Phys. J. C 23 (2002) 455.

[6] G. Ingelman et al., Comput. Phys. Cummun. 101 (1997) 108; T. Sjöstrand, Comput. Phys. Cummun. 82 (1994) 74; A. Hillenbrand (for the HERMES Collaboration), Proc. of the $11^{\text {th }}$ International Workshop on Deep Inelastic Scattering, St. Petersburg, Russia, April 2003.

[7] HERMES Coll., A. Airapetian et al., hep-ex/0605108; submitted to Phys. Rev. Lett.

[8] M. Vanderhaeghen et al., Code for the calculation of DVCS and BH process, Private Commun., 2001.

[9] M. V. Polyakov and C. Weiss, Phys. Rev. D 60 (1999) 114017.

[10] W.-D. Novak, Proc. of HadronPhysics I3 Topical Workshop, 2004; hep-ex/0503010.

[11] Z. Ye (for the HERMES Collaboration), Proc. of the International Europhysics Conference on High Energy Physics, Lisboa, Portugal, July 2005; hep-ex/0512010.

[12] F. Ellinghaus et al., Eur. Phys. J. C 46 (2006) 729.

[13] K. Göeke et al., Prog. Part. Nucl. Phys. 47 (2001) 401.

[14] M. Göckeler et al., Phys. Rev. Lett. 92 (2004) 042002.

[15] Z. Ye (for the HERMES Collaboration), Proc. of the 14th International Workshop on Deep Inelastic Scattering, Tokyo, Japan, April 2006; hep-ex/0606061.

[16] J. Collins et al., Phys. Rev. D 56 (1997) 2982; A. Freund, Phys. Rev. D 61 (2000) 074010.

[17] M. Diehl, Phys. Rept. 388 (2003) 41.

[18] C. Hadjidakis et. al. (for the HERMES Collaboration), Proc. of the 12th International Workshop on Deep Inelastic Scattering, Strbske Pleso, Slovakia, April 2004; hep-ex/0405078

[19] M. Vanderhaeghen et al., Phys. Rev. D 60 (1999) 094017.

[20] HERMES Coll., A. Airapetian et al., Phys. Lett. B 599 (2004) 212.

[21] B. Lehmann-Dronke et al., Phys. Lett. B475 (2000) 147. 